\section{The chosen child}

\section{ThomasBaldwin}

\section{Choosing Children:The Ethical D ilemmas of $G$ enetic Intervention \\ by Jonathan G lover \\ 0 xford U niversity Press, 0 xford, UK \\ $128 \mathrm{pp}, \$ 16 / £ 10$ \\ ISBN 019929092X}

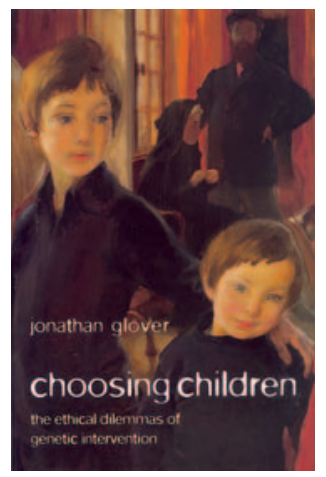

To what extent should parents be allowed to use genetic interventions in order to choose the kind of children they have? Contemporary practice is that using genetic techniques is acceptable to avoid having a child who would inherit a life-threatening or seriously disabling disorder, but unacceptable to enhance the abilities of an otherwise healthy child. Avoiding something bad is allowed, but creating something better is not. In his stimulating new book, Choosing Children: The Ethical Dilemmas of $\mathrm{G}$ enetic Intervention, Jonathan Glover argues that this negative/positive contrast cannot withstand critical scrutiny.

The author starts by discussing the position that interventions enabling parents to avoid having children with inherited disabilities are an unacceptable eugenic policy. This is the best part of Choosing Children, as G lover takes considerable care to ensure that the discussion is informed by a fair representation of the views of the disabled. The uninformed 'common sense' presumption - that life with a serious disability is bound to be irredeemably second-rate in comparison to a life without disabilityneeds to be challenged. Someone who has lost their sight can reorganize their life in such a way that they find new sources of meaning and value; and giving sight to someone who has been blind from birth can be a destructive change that cuts them off from their own sense of identity. Yet, Glover continues, even when all this is acknowledged, in general, disability is not a condition to be welcomed: to be disabled is to lack an ability which contributes to our 'normal' ways of living well and it would be perverse to think that one was going to make life better for a child by making it disabled in some way. The case against using genetic techniques to avoid having a child with an inherited disability is that their use encourages the attitude that the disabled should never have been allowed to exist. However, Glover argues, this association need not be made. There is a standing obligation for all of us to oppose prejudice towards the disabled; but, like those who seek to minimize accidents which cause disabilities, those who seek to avoid having a disabled child do not exemplify this prejudice.

In arguing that this kind of genetic intervention should be allowed, Glover recognizes that he is arguing for a form of 'reproductive autonomy' and, in the latter half of Choosing Children, he discusses what ethical constraints, if any, there should be on the exercise of this freedom. His answer, briefly, is the classic liberal response: that as long as no one is thereby harmed, there is no good reason to limit this freedom. But what about a case in which a couple choose to have a child with an inherited disability? In such a case, the parents are not directly harming the child because its disability is inherited. Glover argues that the requirement to do no harm implies that any such child's inherited disability should not be such that there is a serious risk that its life is not worth living, but beyond this implication, he maintains, no further constraint is warranted. I am not persuaded. I believe that it is wrong to create a child with an unnecessary disability.
Such an act, I agree, is not a direct method of harm, but then I also reject the thesis that doing no harm is all that matters: when one can readily avoid doing so, one should not bring people into existence in such a way that their ability to live well is curtailed by disability. Plainly, more needs to be said about this topic, but Glover's discussion of the issue is surprisingly limited.

In the last part of Choosing Children, Glover considers the application of his liberal principle of reproductive autonomy to hypothetical possibilities of genetic enhancement. He argues that egalitarian anxieties about the use of such techniques for enhancement in a society al ready divided by wealth and other goods are serious, but that, as with existing inequalities concerning educational opportunities, it is possible for societies to regulate these matters if they so wish. The trouble lies not with techniques for enhancement-genetic or educational-but with a society's commitment to equality. In the closing pages of the book, Glover then asks whether a Brave New World of genetic enhancement is something to be feared or enthusiastically anticipated; his answer is "optimism in principle, caution in practice". The grounds for caution are obvious, but the reason he gives for optimism is surprising-namely that we might be able to "expand human intellectual capacity" by some genetic "redesign". To me this sounds too much like a new genetic master race to be a cause for optimism. In my view, we should think, as ever, of the comparison with education: in both fields, modest enhancements are indeed to be welcomed-but we should avoid grand programmes for the redesign of human nature at all costs. That way madness liesboth literally and figuratively.

\section{ThomasBaldwin isa professor in the Department of Philosophy at the University of York, UK. \\ E-mail: trb2@york.ac.uk}

doi:10.1038/sj.embor.7400744 